\title{
Dificuldades Relatadas por Cuidadores de Crianças e Adolescentes com Leucemia: Alterações comportamentais e familiares
}

\author{
Marina Kohlsdorf \\ Áderson Luiz da Costa Junior \\ Universidade de Brasília
}

\begin{abstract}
RESUMO
O tratamento de câncer na infância expõe pacientes e familiares a diversas exigências e desafios, que envolvem alterações na dinâmica familiar, expectativas em relação ao tratamento, medo de recaídas, custos financeiros e mudanças na rotina profissional e familiar. $\mathrm{O}$ objetivo deste estudo foi analisar o impacto do semestre inicial de tratamento para leucemia sobre a rotina, dinâmica familiar, práticas educativas parentais e padrões comportamentais de pacientes e de cuidadores, a partir de entrevistas semiestruturadas realizadas em dois momentos durante o tratamento. Participaram deste estudo 30 cuidadores de crianças e adolescentes em tratamento para leucemia. Os resultados indicam alterações importantes no comportamento dos pacientes, tais como agressividade exacerbada e não-colaboração com tratamento. As práticas educativas parentais foram caracterizadas por maior permissividade, ganhos secundários e superproteção. Por outro lado, os cuidadores indicaram a aquisição gradual de habilidades para lidar com procedimentos médicos-invasivos e efeitos colaterais da medicação antineoplásica, ao longo do tratamento. Os participantes destacaram alterações importantes na rotina profissional dos cuidadores e na rotina social-familiar. Os dados indicam a necessidade de investigar de forma específica e sistemática as dificuldades às quais cuidadores e pacientes são expostos ao longo do tratamento, a fim de possibilitar estratégias de intervenção psicossocial mais eficientes.
\end{abstract}

Palavras-chave: câncer pediátrico; leucemia pediátrica; cuidadores.

\section{ABSTRACT \\ Difficulties Reported by Caregivers of Children and Adolescents With Leukemia: Behavioral and family changes}

Childhood cancer treatment exposes patients and their families to several demands and challenges, which involve changes in family dynamics, beliefs related to the treatment, fear of relapse, financial costs, and changes in both professional and family routines. The main goal of this study was to analyze the impact of the first six months of leukemia treatment upon routine, family dynamics, educational parenting patterns, and patients' and caregivers' behavioral patterns, using semi-structured interviews carried out at two times during treatment. Thirty caregivers participated in the study. The results indicate relevant changes related to patients' behavior, such as heightened anger and absence of collaboration. Educational parenting patterns were characterized by higher permissiveness during this stage in addition to secondary gains and overprotective behavior. On the other hand, the caregivers indicated the gradual acquisition of skills to deal with invasive procedures and side effects related to antineoplasic medication, during treatment. The participants highlighted relevant changes related to caregivers' professional and social-family routine. These data indicate the need to investigate, in a specific and systematic way, the difficulties to which caregivers and patients are exposed to during treatment, so as to promote more efficient psychosocial intervention strategies.

Keywords: pediatric cancer; pediatric leukemia; caregivers.

O câncer na infância corresponde a um grupo de mais de 200 doenças, de etiologia multifatorial, cuja particularidade está na alteração no processo de divisão celular e multiplicação desordenada de células.
Seu tratamento representa diversos desafios tanto para os profissionais de saúde quanto para familiares e pacientes. Modificações de rotina de vida, ansiedade pela resposta clínica ao tratamento, crenças disfuncio- 
nais sobre a patologia, mudanças e desorganizações da dinâmica familiar são comumente vivenciadas por pacientes e familiares (Costa Junior, 2001, 2005).

A criança ou adolescente que vivencia um episódio de câncer precisa lidar com repetidos procedimentos médicos invasivos, efeitos colaterais da medicação, interrupção da rotina escolar, suspensão de algumas atividades de lazer, alteração da dieta, mudanças na auto-imagem e autoconceito, sentimentos de incerteza em relação à evolução do tratamento, dor e perdas que prejudicam a manutenção de relacionamentos pessoais e sociais (Gomes \& cols., 2004; McGrath, Paton \& Huff, 2005; Shiota, Santos \& Miyazaki, 2004).

Para os irmãos da criança ou adolescente em tratamento, as alterações familiares também podem provocar efeitos adversos, que podem levar a diminuição do rendimento escolar, sofrimento decorrente da separação durante períodos de internação, desajustamento psicossocial, sintomas somáticos, sentimentos de rejeição, isolamento, ciúmes, ansiedade, tristeza e incertezas; por outro lado, os irmãos podem também se envolver nos cuidados ao paciente doente, colaborando com a manutenção doméstica e fornecendo suporte emocional a outros membros familiares (Costa \& Lima, 2002; McGrath \& cols., 2005).

Os cuidadores de uma criança ou adolescente em tratamento onco-hematológico, além de acompanharem o paciente, precisam adaptar rotinas sociais, profissionais e familiares, lidar com ameaças de recaídas, medos e expectativas e ainda zelar pelo bem-estar do filho em tratamento, com atenção à medicação, intercorrências, efeitos colaterais, consultas, internações e exames invasivos, eventos que expõem os cuidadores a condições potencialmente estressantes, com implicações adversas à qualidade de vida (Clarke, 2004; Clarke \& Fletcher, 2003; Kars, Duijnstee, Pool, Delden, \& Grypdonck, 2008; McGrath \& cols., 2005; Shiota \& cols., 2004; Silva, Pires, Gonçalves \& Moura, 2002).

De modo geral, o tratamento do câncer pediátrico tem um impacto adverso sobre a rotina social e profissional dos pais, exigindo muitas vezes que deixem o emprego, dedicando mais atenção ao paciente em tratamento (Björk, Wiebe \& Hallström, 2005; Cohn, Goodenough, Foreman \& Suneson, 2003; Dockerty, Skegg \& Williams, 2003; Kerr, Harrison, Medves \& Tranmer, 2004; Steffen \& Castoldi, 2006; Young, Dixon-Woods, Findlay \& Heney, 2002). A atenção concentrada sobre o filho doente pode contribuir para gerar conflitos maritais e prejuízos à comunicação e autocuidado dos pais (Costa \& Lima, 2002; James \& cols., 2002; Steffen \& Castoldi, 2006).

Muitas vezes, o sofrimento da criança e as exigências de cuidados podem acarretar ganhos secundários ao tratamento (vantagens práticas decorrentes de modificações em interações sociais ou práticas educativas), na medida em que o sistema de cuidados parentais é modificado pelas dificuldades em lidar com sentimentos de impotência e frustração em proteger o filho dos procedimentos dolorosos e invasivos, originando alterações de limites, diminuição de responsabilidades, menor autonomia, maior permissividade e superproteção (Herman \& Miyazaki, 2007; McGrath, 2001; Palmer \& cols., 2000; Woodgate, 2004; Young $\&$ cols., 2002).

Torna-se importante destacar que pais e mães deparam-se com demandas distintas advindas do tratamento e costumam lidar diferentemente com os desafios: geralmente as mães assumem o papel de cuidador primário da criança ou adolescente, enquanto os pais atuam como provedores da família (Svavarsdottir, 2005). Para as mães, o acompanhamento do filho ganha papel primordial, muitas vezes levando ao afastamento do emprego. Os desafios concentram-se na tomada de decisões sobre o tratamento, administração de remédios, promoção de conforto e suporte para a criança ou adolescente e irmãos saudáveis, planejamento de atividades familiares, manejo de problemas comportamentais dos filhos, atenção a episódios de febre e outras intercorrências e supervisão de responsabilidades domésticas cotidianas (McGrath, 2001; Svavarsdottir, 2005).

Para os pais, cria-se um conflito entre manter-se trabalhando para o sustento da casa ou permanecer com o paciente, além de demandas de suporte emocional à esposa e filhos. Muitas vezes, os pais têm tendência a se distanciar da situação, enquanto as mães tendem a se envolver emocionalmente (McGrath, 2001; Svavarsdottir, 2005). A partir das modificações nos papéis parentais exigidas pelo tratamento oncohematológico pediátrico, os pais podem vivenciar mudanças em seus relacionamentos conjugais. $\mathrm{O}$ tratamento pode alterar o padrão de funcionamento familiar, modificar a divisão de cuidados parentais e promover o distanciamento físico durante as internações, aumentando a incidência de conflitos maritais. A nova situação de vida pode provocar o surgimento de novos conflitos ou exacerbar conflitos preexistentes (McGrath, 2001; Steffen \& Castoldi, 2006). 
Estudos sobre mudanças e satisfação na relação marital ao longo do tratamento têm obtido resultados distintos. Enquanto alguns casais não relatam modificações em suas relações conjugais, outros pais relatam o fortalecimento das relações maritais e familiares, aumento da coesão familiar, melhorias na comunicação e confiança e atitudes mais positivas em relação ao cônjuge (Beltrão, Vasconcelos, Pontes \& Albuquerque, 2007; Lavee \& Mey-Dan, 2003; Sloper, 2006). Por outro lado, há relatos de enfraquecimento do relacionamento marital e de diminuição na satisfação conjugal, com prejuízos à qualidade da interação sexual e ao sistema de comunicação do casal (Hoekstra-Weebers, Jaspers, Kamps \& Klip, 1998; Lavee \& Mey-Dan, 2003; Pai \& cols., 2007). A satisfação marital parece relacionar-se a diversos fatores relevantes: evolução do tratamento, divisão e organização das tarefas domésticas e cuidados dos filhos, rede de suporte social e familiar e, ainda, disposição e coesão familiares anteriores ao diagnóstico (Dockerty, Williams, McGee \& Skegg, 2000; Hoekstra-Weebers \& cols., 1998).

No caso das leucemias, o semestre inicial de tratamento é crítico e pode implicar alterações psicológicas importantes, tais como incertezas, choque emocional ao diagnóstico e alternância entre os estados de bem-estar e os de medo, tristeza e preocupações com a evolução da patologia (McGrath, 2002). Além disso, os seis primeiros meses concentram internações mais longas e terapêutica antineoplásica mais agressiva, contribuindo para alterações de rotina familiar e mobilização imediata de recursos dos cuidadores. Tornam-se necessárias, portanto, pesquisas de acompanhamento que investiguem alterações das práticas parentais e dificuldades enfrentadas pela família, nesta fase do tratamento.

Considerando algumas das modificações comportamentais e familiares geradas pela exposição ao tratamento do câncer na infância, indicadas na literatura, o objetivo deste estudo foi analisar o impacto dos primeiros seis de tratamento de leucemia sobre a rotina, dinâmica familiar e práticas educativas parentais, além das principais alterações em padrões comportamentais tanto de pacientes quanto de cuidadores.

\section{MÉTODO}

\section{Participantes}

Participaram do estudo 30 cuidadores de crianças ou adolescentes diagnosticados com leucemia: 23 mães, cinco pais, uma tia e uma avó. A média de idade dos cuidadores foi 33,1 anos $(\mathrm{DP}=9,4)$ e a escolaridade variou entre o segundo ano do ensino fundamental e o ensino superior completo. Entre os participantes, 21 eram casados ou viviam em união estável, seis eram divorciados ou separados, dois eram solteiros e um, viúvo. Os pacientes tinham idades entre nove meses e 17 anos (um bebê menor de um ano, 17 entre um e quatro anos, quatro pacientes entre cinco e nove anos, cinco pacientes entre dez e 14 anos e três maiores de 15 anos). Oito participantes eram procedentes de estados da Federação diferentes daquele onde se realizou o trabalho e, em sete casos, o paciente não tinha irmãos.

\section{Instrumentos}

Um roteiro semiestruturado de entrevista foi elaborado para compreender: (a) mudanças pessoais relatadas pelos cuidadores; (b) modificações em padrões comportamentais do paciente; (c) alterações na rotina e dinâmica familiares. Exemplos de questões incluíram: "Você acha que ocorreu alguma mudança em relação ao tratamento? O que causou esta(s) mudança(s)?" e ainda "O que, no tratamento, ficou mais fácil? $\mathrm{O}$ que ficou mais difícil? $\mathrm{O}$ que levou a essa mudança?".

\section{Local}

O estudo foi realizado no Núcleo de OncoHematologia Pediátrica do Distrito Federal, no Hospital de Base do Distrito Federal (HBDF) e do Hospital de Apoio de Brasília (HAB), unidades da Secretaria de Estado da Saúde do Distrito Federal (SES/DF).

\section{Cuidados éticos}

O projeto de pesquisa foi submetido ao Comitê de Ética em Pesquisa (CEP), da Faculdade de Ciências da Saúde da Universidade de Brasília (UnB), sendo aprovado em 22 de dezembro de 2006, sob o Protocolo 142/2006. Todos os participantes, ao serem convidados para participar do estudo e após explicação prévia sobre os objetivos e metodologia da pesquisa, assinaram o Termo de Consentimento Livre e Esclarecido em duas vias. As gravações em áudio das entrevistas foram destruídas e as transcrições dos dados permanecem em arquivo protegido por senha.

\section{Procedimento}

A pesquisa foi organizada em duas fases. A primeira ocorreu cerca de dois a três meses após o início do tratamento $(M=68$ dias; $D P=15,15)$, quando os 
participantes foram entrevistados pela primeira vez, para investigar mudanças desde o início do tratamento. A segunda fase foi realizada após cinco a seis meses ( $M=163$ dias; $D P=16,20)$ desde o início do tratamento, quando os participantes foram solicitados a responder o mesmo roteiro de entrevista, para investigar mudanças ocorridas desde a primeira fase. Todas as entrevistas foram gravadas em áudio e realizadas por um mesmo entrevistador. O momento da primeira e segunda entrevistas variou em função do cumprimento do protocolo de quimioterapia, assim todas as entrevistas foram realizadas na mesma fase de tratamento para todos os familiares.

\section{Análise dos dados}

Para análise de frequência de relatos e seus respectivos conteúdos, as gravações das entrevistas foram transcritas para um banco eletrônico de dados e, posteriormente, organizadas em áreas temáticas e categorias funcionais, de acordo com as proposições teóricas de Bardin (1977/2004) sobre descrições analíticas de conteúdo. Tais relatos foram submetidos a cálculo de frequência para comparação entre os dois momentos do estudo.

\section{RESULTADOS}

Os relatos foram categorizados, segundo seu conteúdo, em duas áreas temáticas: (a) mudanças relatadas pelo cuidador (mudanças em padrões de comportamento do cuidador e paciente, alterações em práticas educativas e afetivas, mudanças no sistema de cuidados parentais, alterações em percepções sobre a saúde do paciente, procedimentos invasivos e elementos médicos hospitalares, mudanças na rotina pessoal e familiar); e (b) relatos do cuidador sobre o impacto do tratamento para a família (mudanças na dinâmica familiar, suporte social proveniente de parentes e mudanças no relacionamento conjugal).

\section{Primeira etapa do estudo: o impacto do diagnóstico do câncer pediátrico}

\section{Área 1: mudanças relatadas pelo cuidador}

\section{Categoria: mudanças em padrões de comportamento}

Em relação às mudanças comportamentais infantis destacadas como benéficas ou satisfatórias, sete cuidadores referiram maior colaboração com o tratamento ("ela, no começo, ficou bem mal humorada, xingava todo mundo, não queria saber de ninguém.
Ficou muito agressiva, não queria remédio, o médico vinha pra examinar ela e ela tirava, foi difícil. Hoje ela não fica mais nervosa" - criança de nove anos). Três participantes relataram maior afetividade em relação aos familiares ("Ah, depois do problema, ela é mais agarrada com a família, bastante carinhosa"). O maior autocontrole por parte do paciente foi referido por três mães ("eu tô achando que ela era muito agressiva, muito agitada, agora já tá mais calma") e duas cuidadoras ressaltaram a retomada de atividades lúdicas como uma mudança importante ("antes ela não tava brincando e agora ela já brinca, já corre" - criança de três anos).

Os acompanhantes também destacaram mudanças comportamentais infantis referidas como insatisfatórias. Três participantes descreveram a ausência de colaboração com o tratamento ("no hospital, ela fica agressiva, mandona, autoritária") e nove cuidadores ressaltaram maior ocorrência de agressividade e/ou menor autocontrole em geral ("ele tá muito agressivo, não me atende") e houve três relatos de expressão de tristeza ou choro por parte do paciente. Uma mãe destacou a maior dependência por parte da criança ("agora só quer ficar no colo, precisa mais da atenção da gente" - paciente de três anos).

Mudanças de comportamento do cuidador, designadas como benéficas ou satisfatórias, foram referidas nesta etapa. Cinco participantes destacaram a oportunidade de aprendizagem em relação à doença ("fiquei muito agitada, porque não sabia nada da doença, agora tô tranquila, já sei um pouco"). Maior atenção e/ou cuidado aos filhos foi ressaltada por quatro cuidadores ("eu cuidava bem dela, só que devido ao problema eu cuido mais"). Oito participantes descreveram maior autocontrole e menores manifestações de ansiedade e medo ("no começo eu fiquei com muito medo, agora não fico tão nervosa nem choro muito") e três cuidadores referiam uma melhoria na qualidade da alimentação, a partir de exigências do tratamento. Cinco participantes destacaram, ainda, sua conformação com a doença e a aceitação do tratamento ("aceitação. Era muito difícil no início pra mim. Eu não aceitava. Mas hoje eu estou aceitando mais").

Em relação às mudanças comportamentais do cuidador referidas como insatisfatórias, três participantes relataram maiores níveis de nervosismo, ansiedade e tensão. Uma mãe relatou sentir medo ("para mim é apavorante, eu estou apavorada") e outra mãe destacou sua tristeza pelo tratamento do filho ("tanta mãe sofrendo, tantas mortes acontecendo. Eu me sinto cada vez mais triste"). 
Foram descritas, também, algumas modificações nas práticas afetivas e educativas parentais. Quase metade da amostra (13 participantes) referiu maior permissividade e/ou concessão de ganhos secundários ao filho, a partir do tratamento ("agora a gente senta mais, conversa, brinca junto, coisa que a gente não fazia"). Por outro lado, foram categorizados cinco relatos de menor permissividade ("não privava ele de muita coisa e hoje, devido ao tratamento, acaba privando ele de certas coisas, coleguinhas que não vão mais lá em casa") e cinco respostas de ausência de mudança nas práticas parentais ("tem regras, tem limite. Ele tem que cumprir como ele cumpria antes").

Em relação a mudanças no sistema de cuidados parentais, a maioria dos participantes (19 cuidadores) destacou alterações na alimentação, enquanto duas mães ressaltaram que a alimentação não foi modificada ("a alimentação lá de casa é sem sal, a gente come muita verdura, então alimentação não teve problema não"). Aumento no controle de higiene e limpeza foi referido por quatro participantes.

Categoria: mudanças em percepções sobre a saúde da criança ou adolescente e elementos médicos hospitalares

A melhoria no estado de saúde da criança ou adolescente foi referida por 18 cuidadores. Por outro lado, uma participante referiu preocupações pelo fato de o organismo do paciente não ter correspondido ao tratamento ("tem o problema, a primeira fase não foi correspondida, o tratamento dele não foi correspondido"). Lidar com procedimentos médicos foi avaliado como mais fácil por 11 participantes ("era muito difícil ver ele sendo torturado, entre aspas, que é muito doloroso, mas agora não, agora eu consigo segurar firme"). Por outro lado, oito cuidadores relataram maiores dificuldades para lidar com procedimentos (“o dia que ele vai fazer a punção, eu não durmo, é difícil seu filho sendo furado várias vezes").

Em relação a lidar com os efeitos colaterais da quimioterapia, sete cuidadores relataram estar mais fácil do que no início do tratamento ("ele vomitava muito e hoje tá mais fácil") e dois participantes referiram estar mais difícil do que no início do tratamento ("fico desorientada quando ele começa a vomitar sem parar"). A melhoria da comunicação com a equipe médica foi ressaltada por cinco participantes ("tive mais facilidade foi entrar em contato com os médicos, eles conversam muito").

\section{Categoria: mudanças na rotina}

A rotina profissional dos cuidadores foi intensamente alterada. A demissão ou afastamento do emprego foi uma ocorrência destacada por 17 participantes e seis cuidadores relataram a necessidade de alteração do horário de trabalho. Cinco acompanhantes referiram a ocorrência de mudança na rotina escolar da criança ou adolescente.Alterações na rotina pessoal do cuidador também foram enfatizadas. Seis participantes relataram uma redução do tempo para atividades pessoais, em função do tempo dedicado aos cuidados com pacientes ("fica mais difícil é a falta de tempo, parece que o tempo encurtou. Abri mão da minha vida pessoal") e 16 cuidadores destacaram a reorganização no cotidiano de atividades pessoais ("acordar cedo"). Alterações no padrão de sono foram referidas por quatro participantes, outros quatro cuidadores descreveram diminuição em atividades sociais e houve quatro relatos de ausência de alteração na rotina ("tento manter as coisas, fazer o que fazia antes"). A diminuição de atividades sociais do paciente foi abordada por cinco participantes ("era uma menina que saía de manhã, chegava praticamente de noite em casa, todos os dias, de segunda a sexta" - adolescente de 17 anos).

De acordo com os relatos, a rotina familiar foi alterada, com redução ou modificações em atividades de lazer, para dez cuidadores ("'a gente ia ao shopping, ia lanchar, pra casa de parente, agora a gente não pode por causa dela") e houve um relato de aumento do tempo disponível em presença de familiares ("antigamente eu saía mais com as minhas amigas. Hoje não, saio família"). Enquanto dois participantes descreveram a permanência dos cuidados com irmãos saudáveis, 11 cuidadores ressaltaram a necessidade de delegar os cuidados dos filhos a outras pessoas ("nosso outro filho a gente teve que deixar com os outros, inclusive hoje ele tá com uma amiga da gente"). Dez participantes destacaram a diminuição da disponibilidade de tempo com outros filhos como uma dificuldade do tratamento ("não tenho tempo de cuidar do outro filho, em relação a ser mãe pra ele, eu deixo muito a desejar").

\section{Área 2: relatos do cuidador sobre o impacto do tratamento para a família}

Em relação ao impacto adverso do tratamento à família, nove participantes mencionaram aumento da coesão e união familiares. Dez cuidadores referiram a redução da atenção dos pais aos irmãos saudáveis, em decorrência do tratamento ("agora a atenção mais é 
pra ela, eu fico pensando nos outros, no de seis anos, porque com esse tratamento a gente ficou muito grudado nela, ele sente, então eu tenho que lidar com isso, conversar com ele"). Quatro participantes destacaram a separação física e/ou geográfica familiar como um sério impacto ("nós temos uma nenezinha que tomou vacina e teve que ficar fora três semanas, aí quem teve que sair foi o neném e a mãe dele"). O suporte social proveniente de parentes e família estendida também foi abordado. Dois cuidadores enfatizaram a importância da disponibilidade de tal suporte, enquanto seis participantes relataram indisponibilidade do suporte social ("não posso contar com família, só posso contar com a minha filha e comigo mesma").

Em relação a alterações do relacionamento marital, a partir do tratamento, sete participantes destacaram melhorias ou aproximação psicossocial do casal ("a doença veio duma forma que abalou toda a família. E ao invés de acontecer o que parecia ser óbvio, da gente separar, foi quando mais a gente se uniu"). Por outro lado, três cuidadores ressaltaram a deterioração das condições de relacionamento e/ou a falta de apoio do cônjuge ("às vezes eu fico chateada com o que meu marido faz, porque pra ele não mudou nada, eu acho que ele devia ficar um pouco com ela também").

\section{Segunda etapa do estudo: convivendo com o diagnóstico e tratamento}

\section{Área 1: mudanças relatadas pelo cuidador}

\section{Categoria: mudanças em padrões de comportamento}

Nesta etapa, os participantes também referiram modificações benéficas dos comportamentos da criança ou adolescente, especialmente no que se refere à colaboração com o tratamento, mencionadas por sete cuidadores. Outras alterações no padrão de comportamentos, relatadas como satisfatórias, incluíram maior autocontrole, destacado por três participantes e retomada de atividades lúdicas e/ou maior interatividade, referida por seis participantes. Entretanto, quatro participantes destacaram a ausência de colaboração com o tratamento: "a única coisa difícil é que ele está muito nervoso, começa a gritar, chorar. Ele não era assim". Metade da amostra referiu maior ocorrência de agressividade por parte da criança ou adolescente e menor autocontrole e expressões de tristeza e choro, por parte do paciente, foram relatadas por dois cuidadores.

Em relação aos comportamentos do cuidador, algumas mudanças benéficas foram mencionadas.
Cinco participantes destacaram a aprendizagem de informações sobre a doença e três cuidadores falaram sobre a disponibilidade de maior atenção e/ou cuidado com os filhos ("mudei completamente. Aprendi a ser mais carinhoso com meus filhos, demonstrar pra eles o amor que eles merecem ter"). Além disso, maior autocontrole e diminuição nas manifestações de medo e/ou ansiedade foram ressaltados por 11 cuidadores e um participante citou melhorias no padrão alimentar, em função de alterações na qualidade da alimentação para a criança ou adolescente. Conformidade e aceitação da doença foram referidas por três participantes. Poucos relatos foram mencionados acerca de mudanças comportamentais do cuidador percebidas como insatisfatórias: menor autocontrole, maior ansiedade, nervosismo e tensão foram destacados por cinco participantes e houve um relato de expressões de tristeza.

Os participantes também mencionaram mudanças nas práticas afetivas e educativas. Dez cuidadores referiram maior permissividade e presença de ganhos secundários ("antigamente, ninguém queria ficar com ele, que ele era impossível, agora todo mundo quer ficar com ele, você trata melhor, paparica mais") e sete participantes destacaram menor permissividade ("não deixo sair pra canto nenhum"). Em relação ao sistema de cuidados parentais, mudanças na alimentação foram ressaltadas por 13 participantes e três cuidadores referiram ausência de modificações na alimentação ("a gente lá em casa já comia sem sal, sem gordura, alimentação já era bem balanceada, não mudou nada"). Alterações no padrão de higiene e limpeza foram destacadas em dois relatos.

Categoria: mudanças em percepções sobre a saúde da criança ou adolescente e elementos médicos hospitalares

Nesta etapa, 23 participantes destacaram a melhoria no estado de saúde aparente da criança ou adolescente ou ainda a ausência de intercorrências. Lidar com procedimentos médicos foi assinalado como mais fácil por 13 cuidadores ("era bastante difícil, ele não entende muito bem o que está acontecendo, pra ele era uma tortura! Hoje ele foi sozinho pra sala de triagem, tirou o sangue, normal, não chorou"). Entretanto, verificaram-se seis relatos de dificuldades crescentes em lidar com tais procedimentos ("ficou mais difícil ver ela ser picada toda vez, porque cada vez mais ela é picada, mais difícil é"). Cinco cuidadores referiram melhoria no modo como lidavam com efeitos colaterais da quimioterapia e apenas um participante avaliou que lidar com efeitos colaterais estava mais difícil. A 
melhoria na comunicação com a equipe médica foi destacada por cinco participantes ("os médicos, pelo menos agora, eles explica melhor pra gente, né? No começo ninguém explicava nada e eu ficava perdida. Agora se eu não sei, eu pergunto, corro atrás, quero saber pra que serve").

\section{Categoria: mudanças na rotina}

Nesta fase final do estudo, dez cuidadores ainda destacaram os prejuízos relativos ao afastamento do emprego, enquanto seis referiram alterações no horário profissional e quatro mencionaram a retomada da rotina escolar pela criança ou adolescente ("voltou pra escola, tá tendo aula"). Em relação à rotina pessoal do cuidador, mais da metade da amostra (16 indivíduos) referiu a manutenção de tempo reduzido para atividades pessoais, em função do tempo dedicado a cuidados com o paciente: "deixei de viver minha vida pra viver a dele". Por outro lado, quatro participantes descreveram a melhoria nas condições de rotina pessoal e houve um relato de melhoria no padrão de sono, enquanto dois cuidadores destacaram a permanência de alterações no padrão de sono.

A rotina social e familiar ainda apresentou alterações importantes. Sete participantes destacaram redução ou modificação de atividades de lazer ("a gente não sai mais") e apenas um cuidador referiu a retomada gradual da rotina social e familiar anterior ao diagnóstico ("a partir desse mês a médica já liberou pra ficar num lugar mais afastado de tumulto, tá mais liberado"). A necessidade de delegar o cuidado de irmãos saudáveis a outras pessoas foi ressaltada por nove participantes (“dói muito deixar uma criança, eu tenho que deixar meu outro filho com vizinho, sei que cuida, mas dói. Meu filho pequeno ainda mamava, ele não tinha nem um aninho, isso me abalou mais" e ainda "levava o neném pra casa da minha mãe, deixou de mamar cedo") e três cuidadores enfatizaram a redução da disponibilidade de tempo com irmãos sau- dáveis ("o que ficou mais difícil foi a criação sobre os meus filhos, fico muito ausente" e também "às vezes eu não tenho tempo com o outro [irmão], tô sempre ausente com o outro, ele é pequenininho, tem 1 ano e 6 meses, precisa muito ainda de mim, fico pensando que não tô cumprindo o meu papel de mãe com ele").

\section{Área 2: relatos do cuidador sobre o impacto do tratamento para a família}

Algumas mudanças na dinâmica familiar foram descritas pelos participantes na segunda etapa do estudo. A maior coesão ou união familiar foi referida por 14 participantes ("o que mudou foi que a gente ficou mais unidos, com esse tratamento"). Nove cuidadores ressaltaram a redução da disponibilidade de atenção a irmãos saudáveis ("um dos meus filhos ficou muito 'assim' no colégio, porque eu não tava junto") e houve um relato sobre a ocorrência de aumento de conflitos com irmãos saudáveis. A separação física e/ou geográfica da família foi destacada por sete cuidadores ("difícil é que eu tô sempre aqui e não fui mais em casa. Muda geral porque você está num Estado e vem pra outro, eu só sei notícias deles pelo telefone"). A importância da disponibilidade de suporte social proveniente de parentes e família estendida foi ressaltada por 17 participantes, enquanto oito cuidadores referiram a indisponibilidade de tal suporte ("eu tenho família aqui, mas pra mim é o mesmo que não tivesse").

Em relação às mudanças no relacionamento marital, quatro participantes destacaram uma aproximação mútua ("eu acho que mais companheirismo de mim com o pai dele") e houve três queixas de falta de apoio conjugal e/ou deterioração do relacionamento ("agora dentro da minha casa sempre tem muita briga, eu e meu esposo a gente briga muito"). A Tabela 1 mostra as frequências de relatos por área e categoria, nas duas fases do estudo: 
TABELA 1

Frequências de Relatos nas Duas Fases de Entrevistas

Frequências de relatos nas entrevistas 1 e 2, por área

Entr.1 Entr.2

Área 1: mudanças relatadas pelo cuidador

$(f)$

$(f)$

1.1) Categoria: mudanças em padrões de comportamento

a) Mudanças no comportamento da criança/adolescente relatadas como satisfatórias

$\begin{array}{cc}10 & 16 \\ 16 & 21 \\ 25 & 23 \\ 5 & 6 \\ 18 & 17 \\ 23 & 15\end{array}$

1.2) Categoria: mudanças em percepções sobre a saúde da criança ou adolescente e elementos médicos hospitalares

a) Mudanças no estado de saúde da criança ou adolescente

$\begin{array}{cc}19 & 23 \\ 19 & 19 \\ 11 & 13 \\ 8 & 6 \\ 9 & 6 \\ 7 & 5 \\ 2 & 1 \\ 5 & 5\end{array}$

1.3) Categoria: mudanças na rotina

Alterações na rotina profissional do cuidador

Alterações na rotina escolar

$\begin{array}{cc}23 & 16 \\ 6 & 4 \\ 30 & 18 \\ 5 & 2 \\ 31 & 16\end{array}$

Área 2: relatos do cuidador sobre o impacto do tratamento para a família

Alterações na rotina pessoal do cuidador

Alterações na rotina social da criança ou adolescente

Mudanças na rotina social-familiar

a) Mudanças na dinâmica familiar

b) Suporte social proveniente de parentes e família estendida

$8 \quad 17$

c) Mudanças no relacionamento marital

10

\section{DISCUSSÃO}

Os relatos obtidos nas entrevistas permitem destacar informações relevantes. Embora os cuidadores tenham relatado mudanças no comportamento da criança, ou adolescente, designadas como benéficas ou satisfatórias, mais da metade da amostra referiu alterações importantes designadas como insatisfatórias, em especial relativas à colaboração com o tratamento e agressividade exacerbada, dado presente na literatura (Gomes \& cols., 2004; McGrath \& cols., 2005; Shiota $\&$ cols., 2004). Os estudos de Gomes \& cols. (2004), McGrath \& cols. (2005) e Shiota \& cols. (2004) também destacaram relatos de cuidadores pediátricos em relação a mudanças comportamentais nos pacientes, enfatizando agressividade, isolamento e dificuldades para cooperar com exames e medicação. Destaca-se, portanto, a importância de intervenções psicossociais com pacientes e familiares para administrar mudanças em padrões de comportamento infantil influenciadas pelo tratamento que possam prejudicar ou dificultar a atuação da equipe e mesmo o próprio repertório de autocuidados do paciente.

As alterações em práticas educativas parentais, caracterizadas por maior permissividade (em decorrência de recomendações do tratamento efetuadas pelos profissionais de saúde) combinadas a ganhos secundários, destacam-se nos relatos das duas etapas de entrevista e são dados que corroboram a literatura (Herman \& Miyazaki, 2007; McGrath, 2001; Palmer \& cols., 2000; Woodgate, 2004; Young \& cols., 2002). Alguns 
pais referiram até mesmo a percepção de culpa frente à imposição de limites à criança ou adolescente em tratamento médico. Intervenções educacionais têm sido extremamente eficazes para reduzir comportamentos de superproteção aliados a ganhos secundários (que podem, inclusive, prejudicar a continuidade do processo de desenvolvimento da criança ou adolescente) e, ao mesmo tempo, apresentar aos pais alternativas mais adequadas para a negociação de limites e práticas disciplinares (Herman, 2007). O estudo de Herman (2007), ao promover um programa educativo que possibilitou progressos na forma de cuidadores lidarem com dificuldades em práticas educativas, destacou a importância de intervenções educacionais para reduzir a superproteção e fornecer aos pais alternativas mais adequadas para a negociação de limites.

Em relação a lidar com procedimentos médicos invasivos, na primeira etapa de entrevistas verificou-se um equilíbrio entre relatos sobre melhoria e agravamento da percepção de enfrentamento de tais procedimentos. Já na segunda etapa de entrevistas, a melhoria em lidar com procedimentos médicos invasivos esteve significativamente presente em mais relatos, quando comparada a observações sobre agravamento em lidar com tais procedimentos. Apesar da indicação, a partir desses dados, sobre a conformação gradual a tais condições e a melhoria em lidar com elas, foram obtidas descrições relevantes sobre a manutenção de dificuldades associadas especialmente a procedimentos de punção lombar. indicando que, mesmo que ocorra a melhoria em lidar com a exposição recorrente a situações de procedimentos invasivos de modo geral, a punção lombar continua sendo um procedimento crítico que cria um contexto propício a manifestações de ansiedade, comportamentos não colaborativos com o tratamento e ameaças à qualidade de vida do paciente e do cuidador.

Destaca-se que, de acordo com os relatos, lidar com efeitos colaterais da quimioterapia melhorou ao longo dos primeiros seis meses de tratamento, possivelmente em virtude do planejamento terapêutico (que prevê a diminuição gradual de internações e terapêuticas menos agressivas) aliado a mecanismos de aprendizagem sobre como manejar tais efeitos. Os estudos de Gomes \& cols. (2004), McGrath (2002), McGrath e cols. (2005) e Shiota e cols. (2004) indicaram relatos mais intensos sobre dificuldades em lidar com efeitos colaterais nas primeiras semanas de quimioterapia, e adaptação ao longo do semestre inicial de tratamento. Essas informações destacam a importância de investir no aprimoramento da equipe de saúde, especialmente na aquisição de habilidades de suporte social, recomendação de cuidados, orientações e comunicação, para minimizar o impacto da terapêutica antineoplásica ainda nas primeiras semanas de tratamento e possibilitar uma adaptação mais rápida aos efeitos colaterais. Essas informações destacam a relevância funcional de se investir no treinamento e aprimoramento da equipe médica, tanto na realização de procedimentos invasivos, quanto na comunicação de diagnóstico, estabelecimento de recomendações ao tratamento e para aquisição de habilidades de suporte social instrumental.

As alterações na rotina profissional dos cuidadores também merecem destaque, conforme indicado pela literatura (Björk \& cols., 2005; Kerr \& cols., 2004; McGrath, 2001; Steffen \& Castoldi, 2006; Young \& cols., 2002). A demissão ou afastamento do emprego, somados à redução da jornada de trabalho e ausência do benefício financeiro de auxílio-doença, garantido em lei para pacientes em tratamento onco-hematológico, constituem fatores críticos que prejudicam imensamente a qualidade de vida do acompanhante e do paciente, conforme indicado por este estudo e pela literatura (Cohn \& cols., 2003; Dockerty \& cols., 2003; James e cols, 2002; McGrath \& cols., 2005). Ressalta-se a importância da garantia de direitos trabalhistas extensivos a todos os trabalhadores, para que possam utilizar licenças e afastamentos que não comprometam sua renda familiar mensal. Os estudos de Björk e cols. (2005), Cohn e cols. (2003), James e cols. (2002), Kerr e cols. (2004), McGrath (2001) e McGrath e cols. (2005) também indicam perdas importantes decorrentes de afastamento do emprego, somado ao aumento de gastos relativos a deslocamento, alimentação, tarifas telefônicas, medicação e acomodações. O prejuízo financeiro conseqüente ao tratamento onco-hematológico constitui um fator extremamente relevante para cuidadores pediátricos.

A modificação na rotina escolar da criança ou adolescente, com afastamento de aulas presenciais e/ou aumento de práticas de exercícios domiciliares (que exigem maior monitoramento dos pais), foi referida na etapa inicial do estudo. Na fase final, alguns cuidadores relataram a retomada da rotina escolar por parte da criança ou adolescente, refletindo assim a mudança gradual em internações, medicações e recomendações médicas que tende a ocorrer até o final do primeiro semestre de tratamento. Nessas condições, tornam-se relevantes ações conjuntas entre hospital, família e escola, no sentido de garantir à criança ou adolescente sua plena re-inserção no contexto social-escolar, promovendo, assim, o adequado retorno às atividades anteriores ao diagnóstico com minimização de prejuízos ao curso de desenvolvimento.

Alterações na rotina pessoal do cuidador foram relatos marcantes tanto neste estudo como nos traba- 
lhos de Costa e Lima (2002), James e cols., (2002) e ainda Steffen e Castoldi (2006). Esses autores indicaram importantes alterações no cotidiano, mobilização da rotina diária em função do tratamento e indisponibilidade de tempo para tarefas pessoais em função dos cuidados ao paciente, resultados bastante similares aos dados obtidos no presente estudo. Na última fase desta pesquisa, houve descrições de melhorias na rotina pessoal, principalmente em função do decréscimo de internações previstas, bem como na duração de cada permanência no hospital. Possivelmente, a disponibilidade de suporte social instrumental poderia auxiliar os cuidadores a organizar sua rotina pessoal de forma a diminuir prejuízos advindos de exigências do tratamento, aumentando a qualidade de vida e, consequentemente, possibilitando uma contribuição mais efetiva ao tratamento.

As mudanças na rotina social e familiar também devem ser ressaltadas. Nas duas etapas de entrevistas, houve muitas descrições sobre a redução ou alteração na rotina de lazer familiar. Entretanto, na última etapa, a frequência de relatos referentes a tais questões foi um pouco menor e a retomada gradual da rotina foi referida, a partir da redução das internações, conforme o planejamento terapêutico de cada caso. Cabe ressaltar a diminuição do tempo dedicado a filhos saudáveis, relatada por acompanhantes como um fator importante das alterações na dinâmica familiar e indicado na literatura como uma das dificuldades vivenciadas pelos irmãos da criança ou adolescente em tratamento (Costa \& Lima, 2002; McGrath \& cols., 2005). Um aspecto crítico descrito pelos cuidadores nas entrevistas refere-se a delegar os cuidados de irmãos saudáveis a outras pessoas, indicado pelos acompanhantes como uma das maiores dificuldades do tratamento. De modo semelhante, participantes provenientes de outros Estados da Federação destacaram a separação física da família e dos outros fillhos como uma exigência significativa do tratamento, inclusive por reduzir a rede de suporte social disponível.

Em relação a mudanças na dinâmica familiar, é marcante a alteração, em diversos níveis, da dinâmica familiar. Houve um aumento gradual na frequência de relatos sobre maior coesão e união familiares ao longo das etapas de estudo, o que pode ser analisado como um ganho secundário potencial ao tratamento e indício de ausência de relação direta entre o tratamento onco-hematológico e prejuízos familiares, ou seja, não necessariamente o contexto de tratamento implica aumento de conflitos, conforme também indicado nos estudos de Clarke e Fletcher (2003) e Dockerty e cols., (2000). Considerando a situação marital, no presente estudo alguns participantes descreveram melhoria na dinâmica conjugal, enquanto outros relataram deterioração do relacionamento e falta de apoio do cônjuge. Os estudos de Beltrão e cols. (2007), Hoekstra-Weebers e cols. (1998), Lavee e Mey-Dan (2003), Pai e cols. (2007), Sloper (2006) e Steffen e Castoldi (2006) indicaram resultados similares: para alguns casais, o tratamento representou a reorganização da dinâmica marital com melhorias à comunicação, aumento da confiança interpessoal e da satisfação marital, mas, para outros casos, houve deterioração da dinâmica conjugal, exacerbação de conflitos preexistentes e prejuízos à interação sexual. Tais resultados parecem indicar que não há, a priori, uma relação direta entre o contexto de tratamento e a deterioração do relacionamento conjugal, pois cada casal possui uma dinâmica própria que é influenciada de maneiras diferentes pelo tratamento., dado indicado a partir da análise de diversos estudos sobre tal temática. Além disso, inúmeros outros fatores também atuam nesse contexto, influenciando tanto a relação marital quanto a dinâmica familiar como um todo.

\section{CONSIDERAÇÕES FINAIS}

Considerando os relatos de dificuldades em manter as práticas afetivas e educativas parentais com os irmãos do paciente em tratamento, uma possibilidade de suporte instrumental que poderia ser implementado refere-se à implementação de creches para os filhos de cuidadores, ou ao menos para aqueles filhos que ainda estejam em período de amamentação. Embora a presença de creches em ambiente hospitalar possa aumentar índices de patologias infecto-contagiosas e comprometer indicadores de biossegurança, reconhece-se a importância da amamentação nos primeiros meses de vida da criança. Promover o desmame precoce, como foi apontado por algumas mães como uma exigência do tratamento, pode significar futuramente outros problemas para a saúde da criança, que poderiam ser evitados com a garantia de permanência da criança próxima à mãe durante o período de amamentação.

Além disso, a localização da creche, bem como cuidados com higiene e limpeza, poderia tornar a perspectiva da creche hospitalar uma proposta bastante viável e eficaz em termos de prevenção primária a outras patologias pediátricas, estendida a mães em outros contextos de internação, contribuindo para melhorar as condições de saúde geral da população. Torna-se ainda relevante incentivar a formulação de políticas públicas que visem à melhoria das condições de atendimento no Sistema Único de Saúde, para que 
ações instrumentais possam ser efetivamente instauradas e haja diminuição dos custos do tratamento.

Treinamentos para equipes multidisciplinares de atendimento, desde os currículos de graduação e de cursos técnicos, também devem ser ressaltados, no sentido de instaurar um modelo de prevenção a doenças e promoção de saúde em que diversas abordagens possam contribuir para a diminuição de riscos e danos e aumento da qualidade de vida de familiares de pacientes pediátricos em tratamento onco-hematológico. Especialmente em relação à atuação da equipe médica, torna-se bastante importante investir em treinamento para procedimentos de punção lombar, comunicação de diagnóstico e atuação multidisciplinar, a fim de minimizar situações que propiciem a pacientes e acompanhantes custos comportamentais elevados. Especificamente em relação a procedimentos invasivos e exames de punção lombar, destaca-se a importância do investimento em treinamentos para a equipe, focalizando a comunicação de orientações importantes e o fornecimento de suporte social. A exposição a procedimentos médicos invasivos representa um contexto bastante doloroso para o paciente, com impacto intenso para os cuidadores, e o treinamento da equipe poderia proporcionar uma adaptação mais rápida a este contexto aversivo, mesmo frente a um procedimento repetido e doloroso.

De modo especial, a investigação de quais fatores são preponderantes em cada fase do tratamento e as diferenças de enfrentamento em etapas distintas de quimioterapia, por exemplo, possibilita a investigação sobre como ocorre o processo de adaptação às contingências de tratamento, contribuindo para o desenvolvimento de protocolos de intervenção psicossocial voltados às necessidades dos cuidadores, tornando-os mais ativos e envolvidos com os cuidados de saúde do paciente pediátrico. Para proporcionar um melhor atendimento, suporte e intervenção a familiares de pacientes pediátricos em tratamento, destaca-se, ainda, a necessidade de mais estudos com análises complementares, em âmbito nacional e multicêntrico, que focalizem as estratégias de enfrentamento, o ajustamento psicológico e a adaptação de cuidadores de crianças e adolescentes frente ao diagnóstico e ao tratamento de patologias neoplásicas.

\section{REFERÊNCIAS}

Beltrão, M. R. L. R., Vasconcelos, M. G. L., Pontes, C. M., \& Albuquerque, M. C. (2007). Câncer infantil: Percepções maternas e estratégias de enfrentamento frente ao diagnóstico. Jornal de Pediatria, 83(6), 562-566.

Bardin, L. (2004). Análise de conteúdo (L. A. Reto \& A. Pinheiro, Trad.). Lisboa: Edições 70. (Original publicado em 1977)
Björk, M., Wiebe, T., \& Hallström, I. (2005). Striving to survive: Families' lived experiences when a child is diagnosed with cancer. Journal of Pediatric Oncology Nursing, 22(5), 265-275.

Clarke, J. (2004). Whose power/authority/knowledge? Conundrums in the experiences of parents whose children have cancer. Social Work in Health Care, 40(2), 13-35.

Clarke, J. N., \& Fletcher, P. (2003). Communication issues faced by parents who have a child diagnosed with cancer. Journal of Pediatric Oncology Nursing, 20(4), 175-191.

Cohn, R. J., Goodenough, B., Foreman, T., \& Suneson, J. (2003). Hidden financial costs in treatment for childhood cancer: An Australian study of lifestyle implications for families absorbing out-of-pocket expenses. Journal of Pediatric Hematology and Oncology, 25(11), 854-863.

Costa, J. C., \& Lima, R. A. G. (2002). Crianças/adolescentes em quimioterapia ambulatorial: Implicações para a enfermagem. Revista Latino-Americana de Enfermagem, 10(3), 321-333.

Costa Junior, A. L. (2001). Análise de comportamentos de crianças expostas à punção venosa para quimioterapia. Tese de doutorado não-publicada, Universidade de Brasília.

Costa Junior, A. L. (2005). Psicologia da saúde e desenvolvimento humano: $\mathrm{O}$ estudo do enfrentamento em crianças com câncer e expostas a procedimentos médicos invasivos. Em M. A. Dessen \& A. L. Costa Junior (Orgs.), A ciência do desenvolvimento humano: Tendencias atuais e perspectivas futuras (pp. 171-189). Porto Alegre: Artmed.

Dockerty, J. D., Skegg, D. C. G., \& Williams, S. M. (2003). Economic effects of childhood cancer on families. Journal of Paediatric Child Health, 39(4), 254-258.

Dockerty, J. D., Williams, S. M., McGee, R., \& Skegg, D. C. (2000). Impact of childhood cancer on the mental health of parents. Medical and Pediatric Oncology, 35(5), 475-483.

Gomes, R., Pires, A., Moura, M. J., Silva, L, Silva, S., \& Gonçalves, M. (2004). Comportamento parental na situação de risco do cancro infantil. Análise Psicológica, 22(3), 519-531.

Herman, A. R. S., \& Miyazaki, M. C. O. S. (2007). Intervenção psicoeducacional em cuidador de criança com câncer: Relato de caso. Arquivos de Ciências da Saúde, 14(4), 238-244.

Herman, A. R. S. (2007). Câncer pediátrico: Impacto de intervenção psicoeducacional sobre enfrentamento e práticas parentais. Tese de doutorado não-publicada, Faculdade de Medicina de São José do Rio Preto.

Hoekstra-Weebers, J. E. H. M, Jaspers, J. P. C., Kamps, W. A., \& Klip, E. C. (1998). Marital dissatisfaction, psychological distress, and the coping of parents of pediatric cancer patients. Journal of Marriage and the Family, 60(4), 1012-1021.

James, K., Keegan-Wells, D., Hinds, P. S., Kelly, K. P., Bond, D., Hall, B., Mahan, R., Moore, I. M., Roll, L., \& Speckhart, B. (2002). The care of my child with cancer: Parent's perceptions of caregiving demands. Journal of Pediatric Oncology Nursing, 19(6), 218-228.

Kars, M. C., Duijnstee, M. S. H., Pool, A., Delden, J. J. M., \& Grypdonck, M. H. F. (2008). Being there: Parenting the child with acute lymphoblastic leukaemia. Journal of Clinical Nursing, 17(12), 1553-1562.

Kerr, L. M. J., Harrison, M. B., Medves, J., \& Tranmer, J. (2004). Supportive care needs of parents of children with cancer: Transition from diagnosis to treatment. Oncology Nursing Forum, 31(6), 116-126. 
Lavee, Y., \& Mey-Dan, M. (2003). Patterns of change in marital relationships among parents of children with cancer. Health \& Social Work, 28(4), 255-263.

McGrath, P. (2001). Findings on the impact of the treatment for childhood acute lymphoblastic leukaemia on family relationships. Child and Family Social Work, 6(3), 229-237.

McGrath, P. (2002). Beginning treatment for childhood acute lymphoblastic leukemia: Insights from the parent's perspective. Oncology Nursing Forum, 29(6), 988-996.

McGrath, P., Paton, M. A., \& Huff, N. (2005). Beginning treatment for pediatric acute myeloid leukemia: The family connection. Issues in Comprehensive Pediatric Nursing, 28(2), 97114.

Pai, A. L. H., Greenley, R. N., Lewandowski, A., Drotar, D., Youngstrom, E., \& Peterson, C. C. (2007). A meta-analytic review of the influence of pediatric cancer on parent and family functioning. Journal of Family Psychology, 21(3), 407-415.

Palmer, L. M., Erickson, S., Shaffer, T. Koopman, C., Amylon, M., \& Steiner, H. (2000). Themes arising in group therapy for adolescents with cancer and their parents. International Journal of Rehabilitation and Health, 5(1), 43-54.

Shiota, C. M., Santos, A. R. R., \& Miyazaki, M. C. O. S. (2004). Problemas de comportamento em crianças com câncer: O papel dos pais. Em M. Z. S. Brandão, F. C. S. Conte, F. S. Brandão, Y. K. Ingberman, C. B. Moura, V. M. Silva \& S. M. Oliane (Orgs.), Sobre comportamento e cognição: Vol. 14. Estendendo a psicologia comportamental e cognitiva aos contextos da saúde, das organizações, das relações pais e filhos e das escolas (pp. 261-266). Santo André, SP: ESETec.

Silva, S., Pires, A., Gonçalves, M., \& Moura, M. J. (2002). Cancro infantil e comportamento parental. Psicologia, Saúde \& Doenças, 3(1), 43-60.

Sloper, P. (2006). Needs and responses of parents following the diagnosis of childhood cancer. Child: Care, Health and Development, 22(3), 187-202.

Steffen, B. C., \& Castoldi, L. (2006). Sobrevivendo à tempestade: A influência do tratamento oncológico de um filho na dinâmica conjugal. Psicologia: Ciência e Profissão, 26(3), 406-425.

Svavarsdottir, E. K. (2005). Gender and emotions: Icelandic parents experiencing childhood cancer. International Journal of Nursing Studies, 42(5), 531-538.

Woodgate, R. L. (2004). Cancer symptom transition periods of children and families. Journal of Advanced Nursing, 46(4), 358-368

Young, B., Dixon-Woods, M., Findlay, M., \& Heney, D. (2002). Parenting in a crisis: Conceptualizing mothers of children with cancer. Social Science \& Medicine, 55(10), 1835-1847.

Recebido: 07/09/2009

Última revisão: 03/12/2009

Aceite final: 18/01/2010

\section{Sobre os autores:}

Marina Kohlsdorf: Mestre em Processos de Desenvolvimento Humano e Saúde pela Universidade de Brasília (2008). Doutoranda do Programa de Pós-graduação em Processos de Desenvolvimento Humano e Saúde (PGPDS), Instituto de Psicologia, Universidade de Brasília.

Áderson Luiz da Costa Junior: Doutor em Psicologia pela Universidade de Brasília (2001), Pós-Doutorado pela Universidade Estadual de Campinas (2003). Professor Adjunto no Departamento de Psicologia Escolar e do Desenvolvimento, Instituto de Psicologia, Universidade de Brasília.

Endereço para correspondência: Áderson Luiz da Costa Junior - Universidade de Brasília - Instituto de Psicologia - Departamento de Psicologia Escolar e do Desenvolvimento - Campus Universitário Darcy Ribeiro - ICC - Ala Sul - Asa Norte - 70910-900 Brasília/DF. Endereço eletrônico: aderson@unb.br. 